\title{
Levels of burnout among registrars and medical officers working at Bloemfontein public healthcare facilities in 2013
}

\author{
U Sirsawy ${ }^{\mathrm{a}}$, WJ Steinberg ${ }^{\mathrm{a}, *}$ (D) and JE Raubenheimer ${ }^{\mathrm{b}}$ (D) \\ ${ }^{a}$ Department of Family Medicine, Faculty of Health Sciences, University of the Free State, Bloemfontein, South Africa \\ ${ }^{b}$ Department of Biostatistics, Faculty of Health Sciences, University of the Free State, Bloemfontein, South Africa \\ *Corresponding author, Email:Steinbergwj@ufs.ac.za
}

\begin{abstract}
Background: Burnout is prevalent among medical personnel and affects their work environment. This study investigated the level of burnout among registrars and medical officers at public healthcare facilities in Bloemfontein.

Methods: An analytical cross-sectional study included registrars and medical officers at four public healthcare facilities in Bloemfontein. Socio-demographic information was collected and participants completed the Maslach Burnout Inventory, which consists of three subscales: emotional exhaustion, depersonalisation and personal accomplishment.

Results: Of the 300 potential participants, 205 were included in the result. Only 3.4\% of the participants showed no burnout on all three subscales and $28.3 \%$ had only low to moderate levels of burnout on all three subscales. A quarter (26.3\%) of the participants showed high burnout on one subscale, but not the others. Furthermore, $26.3 \%$ showed a high level of burnout on any combination of two of the three subscales. A high degree of burnout on all three subscales was found in $15.6 \%$ of the participants.

Conclusion: Burnout is a major problem among registrars and medical officers working in public hospitals in Bloemfontein. An action plan needs to be put in place in partnership with the Departments of Health and Higher Education to prevent burnout among an important working cadre.
\end{abstract}

Keywords: Bloemfontein, burnout, Maslach Burnout Inventory, medical officers and registrars, public hospital

\section{Introduction}

Chronic stress among doctors is emotionally draining and poses a significant risk of burnout. ${ }^{1}$ The modern medical environment is stressful, ${ }^{2}$ it is complex and doctors have to continually learn new skills, work long hours, and face death and dying of their patients on a daily basis. Other factors include fatigue, high demand on work/life balance, dealing with emergencies and patient expectations and demands information overload, administration and personality factors. ${ }^{2-4}$ When compared with $18 \%$ in the general working population, stress among caregiving professionals, including doctors and nurses, at $28 \%$ is among the highest of all professions. ${ }^{3-5}$

Olkinuora et al. ${ }^{4}$ stated that burnout is an extreme response to stress and can be defined as'an experience of physical, emotional and mental exhaustion, caused by long-term involvement in situations that are emotionally demanding'. Depending on the duration and severity of the burnout there are often further negative social consequences, which include withdrawal at the workplace (so called 'inner resignation') and negative effects on private life as well as an increased risk of absence from work. Burnout does not only affect the doctors on a personal level. A study by Salyers et al. 7 found that $58 \%$ of doctors felt that burnout had a negative impact on their work quality. Burnout has been linked to lower patient satisfaction and outcomes and perceived quality of care. A literature review by Amoafo et al. ${ }^{8}$ in 2014 found that the prevalence of burnout in doctors is increased by female gender, younger age, long working hours, low job satisfaction and work-home conflict.

In a study done by Cahoon ${ }^{9}$ on 1870 Canadian physicians, $45.7 \%$ were in an advanced phase of burnout. Almost half (47.6\%) of the female physicians reported advanced phase burnout as did $44.6 \%$ of male physicians (the slight difference was not statistically significant). In Mexico City, a study of 312 junior doctors revealed that $40 \%$ showed burnout, which was strongly associated with the working of 12 -hour shifts. ${ }^{10}$

The Maslach Burnout Inventory (MBI), designed by Maslach, Jackson, Leiter, Schaufeli and Schwab, ${ }^{11}$ is a survey assessing burnout in professionals from different sectors. The Maslach Burnout Inventory Human Service Survey (MBI-HSS) was designed for human service workers and has since been expanded to other professions. The MBI-HSS evaluates the degree of burnout using three subscales: emotional exhaustion, depersonalisation and personal accomplishment.

Using the $\mathrm{MBI}$, a cross-sectional survey of family doctors, conducted in 12 European countries, revealed high levels of burnout affecting two-thirds of participants: while 35\% did not report burnout on any of the three subscales, $43 \%$ reported high emotional exhaustion, 35\% reported depersonalisation, 32\% reported a lack of personal accomplishment, and $12 \%$ reported high burnout on all three subscales simultaneously. ${ }^{12}$

Similar studies have been done in South Africa. A study done at Cape Town primary healthcare facilities found that $76 \%$ of the 132 participating medical doctors experienced burnout, as indicated by high scores on either the emotional exhaustion or depersonalisation subscale. ${ }^{13}$ Peltzer et al..$^{14}$ found high levels of burnout in 402 doctors randomly selected from registered medical practitioners. At a primary healthcare facility in the Free State Province, levels of burnout and compassion fatigue were studied in professional nurses involved in the antiretroviral treatment programme: $69 \%$ of 
the participants reported high levels of emotional exhaustion and $85.7 \%$ high levels of depersonalisation. ${ }^{15}$

Junior doctors at Red Cross War Memorial Children's Hospital in Cape Town showed significant levels of burnout, which negatively affected retention skills and quality of care at the hospital. The $\mathrm{MBI}$ was sent to 39 junior doctors. Of the 22 participants, $100 \%$ experienced a high degree of burnout on at least one of the three subscales. Of those surveyed, 95\% expressed an intention to leave the hospital. ${ }^{16}$

As part of the Free State healthcare services, there are four teaching hospitals in Bloemfontein that employ registrars and medical officers (doctors in training) in various fields of specialty. Among this working group there is a constant unhappiness regarding the work-related expectations. Anecdotally, there seems to be a high level of unnecessary interpersonal conflict, especially occurring around referral of patients. The authors were interested in measuring the degree of burnout among this workforce and comparing the results to the degree of burnout in other locations.

During the literature search the authors were able to find limited literature that had measured the level of burnout among South African doctors in training.

This study aimed to measure the level of burnout in registrars and medical officers working at four public healthcare training facilities in Bloemfontein, Free State.

\section{Methods}

An analytical cross-sectional study design was chosen; the research paradigm is quantitative descriptive from a positivistic worldview.

This study took place at four public healthcare facilities in Bloemfontein, Free State and included a tertiary and quaternary level hospital, referral specialist hospital, district hospital, and psychiatric complex. All registrars and medical officers employed by these public healthcare facilities between December 1, 2012 and May 31, 2013 were included in the study population.

Participants had to be registered medical practitioners with the Health Professions Council of South Africa (HPCSA), working as full-time registrars or medical officers. Intern and specialist doctors, doctors working on a locum basis, and doctors working outside the selected public healthcare facilities were excluded.

In total 300 potential participants, 243 registrars and 57 medical officers rotating at the four public healthcare facilities, were included as the study population in the study.

\section{The Maslach Burnout Inventory Human Service Survey}

The MBI-HSS consists of 22 items that examine the three dimensions of burnout: emotional exhaustion (EE), depersonalisation (DP), and personal accomplishment (PA). The dimensions are measured by three subscales, and use a sevenpoint Likert scale response to rate the frequency of each item, starting at $0=$ 'never' up to $6=$ 'every day'.11

The EE subscale combines aspects of physical and mental exhaustion, and includes characteristics like physical wear, exhaustion and loss of energy. The DP subscale manifests negative aspects of the responses and attitudes involving other individuals,
Table 1: Cut-off points for the three dimensions of burnout

\begin{tabular}{lccc}
\hline & \multicolumn{3}{c}{ Dimensions of burnout } \\
\cline { 2 - 4 } Degree of burnout & EE & DP & PA \\
\hline High & $\geq 27$ & $\geq 13$ & $\leq 31$ \\
Moderate & $17-26$ & $7-12$ & $32-38$ \\
Low & $0-16$ & $0-6$ & $\geq 39$ \\
\hline
\end{tabular}

Notes: $\mathrm{EE}=$ emotional exhaustion; $\mathrm{DP}=$ depersonalisation; $\mathrm{PA}=$ personal accomplishment.

mainly co-workers. The PA subscale shows typical negative responses aimed at the respondent and the work itself. These responses are related to depression, low morale, low productivity, inability to withstand pressure and poor self-esteem.

A high degree of burnout is reflected in high scores for the EE subscale ( $\geq 27$ ) and DP subscale ( $\geq 13$ ) and low scores for the PA subscale $(\leq 31)$ as shown in Table 1 . The scores are not combined into a single, total score. ${ }^{11}$

\section{Data collection}

Data were collected through a self-administered questionnaire, consisting of two parts:

- Part A: socio-demographic characteristics, including age, gender, marital status, years since qualification, workplace and working hours (including weekly overtime);

- Part B: MBI-HSS questionnaire.

The validated instruments were purchased from Mind Garden, Inc. ${ }^{11}$ and permission and licence were obtained to utilise the questionnaires in South Africa.

The study was approved by the Ethics Committee of the Faculty of Health Sciences, University of the Free State (ECUFS 129/2012). Written approval from the Chief Executive Officers of all four public healthcare facilities in Bloemfontein was obtained. All participants signed informed consent.

\section{Statistical analysis}

Statistical analysis was done by the Department of Biostatistics, Faculty of Health Sciences, University of the Free State using SAS/ STATC (SAS Institute, Cary, NC, USA) software version 12.3 for Windows. Reliabilities for the MBI-HSS subscales were tested by computing Cronbach's alpha coefficients. Categorical variables were compared using chi-square analyses, and levels of burnout on the various subscales were tested for significant differences according to certain demographic variables using ANOVAs. Pearson correlation coefficients were calculated to compare continuous variables.

\section{Validation of questionnaire instrument}

The MBI-HSS has shown good reliability and validity in the human service professions. In the South African context, research evidence appears to confirm these findings. Basson and Rothmann found that, in terms of reliability, the MBI-HSS seems to be an internally consistent scale when assessing Cronbach's alpha coefficient in various samples. ${ }^{17}$

This study also found good reliability for the three subscales, with alpha coefficients of 0.91 for $E E, 0.74$ for $D P$, and 0.64 for PA. The cut-off points used in this study for the three subscales of the MBI-HSS are shown in Table 1. 


\section{Results}

A total of 205 questionnaires were returned from the 300 eligible participants, giving a response rate of $68.3 \%$.

The age of the participants ranged from 26 to 77 years, with a median age of 32 and an interquartile range (IQR) from 29 to 36. They had graduated from 1 to 30 years previously (with a median of 6 and an IQR ranging from 5 to 9), and had been working in their current position ( $n=193$ ) between 0 and 22 years (median: 2, IQR $1-3)$. It was interesting to note that years of experience seemed to correlate with lower emotional exhaustion $(r=-0.15 ; p=0.04)$, and lower depersonalisation $(r=-0.18 ; p=0.01)$, but did not correlate at all with personal accomplishment $(r=-0.01 ; p=0.88)$.

It was evident that the participants worked long hours: their normal working week was anything from 40 hours (the prescribed workweek) to more than double that, at 91 hours (median 40 hours, and third quartile 46 hours). When describing the hours that they worked in the past week, the range was again from 40 to 90 hours, but now with a median of 45 hours and a third quartile of 51 hours. In addition, the participants indicated that in the past week they had worked up to 96 hours of overtime (with a median of 18 hours and a third quartile of 32 hours), although it may be that some of the participants indicated their total work time, and not only the overtime.

Table 2 shows the demographic profile of the sample: the majority of the participants were male $(61.3 \%)$, white $(59.5 \%)$ and married (67.5\%). Most participants were registrars (81.5\%) working mixed shifts and night duty (83.9\%). This profile was a fairly accurate representation of the registrar and medical officer population of these hospitals.

Only $17(8.3 \%)$ participants had rotated through three of the facilities in the past three months, while most (74.6\%) worked at

Table 2: Demographic data of registrars and medical officers at the four public healthcare facilities in Bloemfontein

\begin{tabular}{lcc}
\hline Factor & $n$ & $\%$ \\
\hline Gender $(n=204)$ & 125 & \\
\hline Male & 79 & 61.3 \\
\hline Female & & 38.7 \\
\hline Ethnicity $(n=205)$ & 58 & \\
\hline Black & 12 & 28.3 \\
\hline Coloured & 7 & 5.9 \\
\hline Indian & 122 & 3.4 \\
\hline White & 6 & 59.5 \\
\hline Other & & 2.9 \\
\hline Marital status $(n=203)$ & 137 & \\
\hline Married & 59 & 67.5 \\
\hline Single & 7 & 29.1 \\
\hline Divorced & 172 & 3.4 \\
\hline Job description at time of study $(n=205)$ & & \\
\hline Registrar & 167 & 81.5 \\
\hline Medical officer & 38 & 18.5 \\
\hline Shift $(n=205)$ & & \\
\hline Day shift & 33 & \\
\hline Mixed shifts and night duty & & \\
\hline & & \\
\hline
\end{tabular}

only one facility. None of the participants rotated at all four public healthcare facilities during the study period.

Figure 1 plots the MBI-HSS values for the sample. The dotted lines show the burnout category boundaries for each subscale, and have been set at the midpoint between the two values at the limit of each respective category (i.e. there are no participants on the lines, only above or below).

Almost half (47.8\%) of the sample (median: 26$)$ suffered from high emotional exhaustion (98 participants in the high EE range), $40 \%$ of the sample showed high levels of depersonalisation (median: 11,82 participants in the high DP range), and $38 \%$ (median: 33 ) of the sample felt a very low sense of personal accomplishment (78 participants in the high-burnout PA range, reporting very low levels of personal accomplishment).

Furthermore, only $65(31.7 \%)$ participants showed moderate to low levels of burnout on all three subscales, while 32 (15.6\%) showed high levels of burnout on all three subscales. A further $86(42.0 \%)$ showed high levels of burnout on any combination of two of the three subscales.

Of further interest was the combination of burnout across subscales - in other words, a doctor experiencing burnout in EE, DP and PA simultaneously could be thought of as being much more burnt out than one experiencing burnout on, for example, only EE but not DP or PA. For the purposes of this comparison, none of the subscales was considered more serious than any other, only the number of burnout types in each category (high, moderate or low) was tallied. Of the 27 possible combinations ( $27=3^{3}$ : high, moderate, and low, spread across EE, DP and PA), a doctor could exhibit one of three main patterns: burnout levels were the same across all three subscales (and then either all three high, moderate or low); evenly spread (one each of high, moderate and low), or a two-one distribution (two subscales being either high, moderate or low, and the third something else). These patterns are reflected in Figure 2.

The groupings are made on the horizontal axis, where the baseline indicates two of the three subscales at that level. The level above that indicates where the third subscale lay. Thus the solid bars in each group represent respondents who scored in that group for all three subscales (note that the solid bar can only be the bar in its group - e.g. only the low bar - as the third subscale - can be solid in the low grouping, where the first and second subscales were already low). The patterned columns indicate how the third subscale for that respondent differed from the first two (which were then also the same). As an example, the left-most column in the first group indicates two low subscales, together with the third subscale on low as well (thus, all three subscales on low burnout, $n=7$ ). The second column indicates that while two subscales were low, the third was moderate $(n=26)$. And the third column indicates that while two subscales were low, one was high $(n=13)$. The evenly spread pattern (one each of high, moderate and low) is on the far right.

From this diagram it is evident that only $3.4 \%$ of the participants showed low burnout on all three subscales, while the largest combination of subscales was shown by the study to be the two most indicative of burnout: high burnout on two subscales and moderate burnout on a third $(n=43 ; 21.0 \%)$, and high burnout on all three ( $n=32 ; 15.6 \%)$. These two groupings represented more than a third of all the participants. In summary, only $28.3 \%$ of the participants had combinations of only low and moderate 


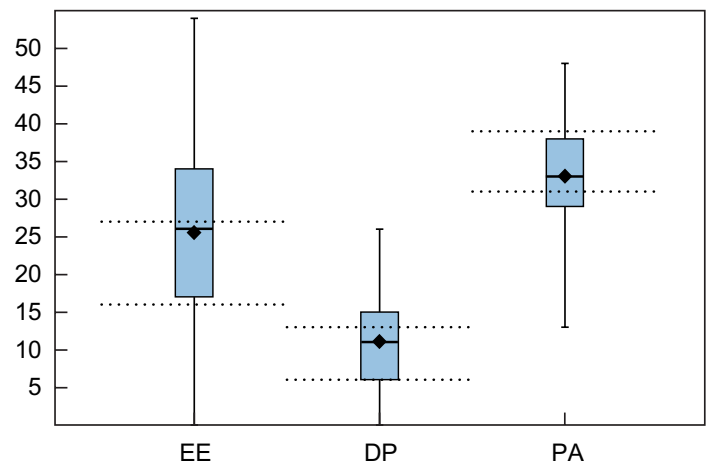

Figure 1: Box plots of MBI-HSS scores (dotted lines show boundaries for low/moderate/high burnout)

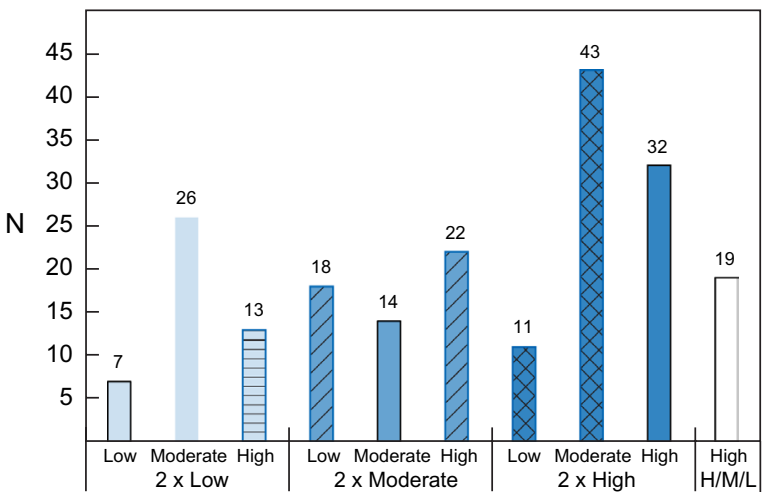

Figure 2: Burnout category combinations.

levels of burnout on all three subscales, and a quarter (26.3\%) of the participants showed high burnout on only one subscale, but not the others. Furthermore, $26.3 \%$ showed a high level of burnout on any combination of two of the three subscales (this includes the $21.0 \%$ mentioned earlier).

Table 3 shows the distribution of the sample across the high, moderate and low levels of burnout for each subscale, for the sample, as well as by gender, ethnicity, jobs roles and shifts. When looking at each subscale it is apparent that overall by far the majority of participants scored high levels of burnout on each of the first two subscales: EE $47.8 \%$, DP $40.0 \%$; while the largest proportions were in moderate (43.4\%) and high (38.1\%) on the PA subscale. Only $29 \%$ or fewer scored low levels of burnout on each of the subscales.

For males, more than a third of the participants showed a high degree of burnout on EE, DP or PA. For females, however, about half showed high burnout on DP, while about two-thirds showed high burnout on $\mathrm{EE}$, and about $40 \%$ each on high and moderate PA burnout. The two genders only differed statistically on $E E$ $\left(\chi^{2}=18.6, \mathrm{df}=2, p<0.01\right)$.

The distribution of burnout levels by ethnic groups also showed no statistically significant differences for EE or PA, although the chi-square value was significant for DP (chi-square $=23.03$, $\mathrm{df}=8, p<0.01$ ), where it would appear that the black participants experienced less depersonalisation than the other ethnic groups.

When comparing registrars with medical officers, no statistically significant differences were found, with both groups experiencing burnout in approximately equal proportions. The levels of burnout for DP and PA were similar for those doctors working day shift vs. mixed shifts. However, those working mixed shifts, which is directly related to hours of work, showed significantly higher levels of $\mathrm{EE}$ (chi-square $=7.32, \mathrm{df}=2, p<0.05$ ).

\section{Discussion}

This study of burnout among doctors in Bloemfontein public hospitals was done by using a validated tool (MBI-HSS) to measure levels of burnout among registrars and medical officers. A high degree of burnout on all three subscales simultaneously was found in $15.6 \%$ of the participants, whereas a mere $31.7 \%$ of the participants had only low to moderate levels of burnout on all three subscales. The remaining $52.7 \%$ showed a high level of burnout on at least one, possibly two, of the three subscales (in fact, exactly $26.35 \%$ showed high burnout either on only one dimension, or on any two dimensions).

These levels of burnout are among the highest reported for medical staff and are almost as high as measured among European family doctors. ${ }^{12}$

More female participants reported high degrees of burnout in the EE category. This could be as a result of female doctors needing to balance more family responsibilities with those of being a medical practitioner. Peltzer et al..$^{14}$ and Amoafo et al. ${ }^{8}$ showed that there was a link between EE and gender, and that female doctors experienced more job stress than male doctors and were at a higher risk of burnout. Other factors, such as similar opportunities to build resilience and expectations to perform better under traditional circumstances, need to be further investigated.

According to Peltzer et al. ${ }^{14}$ white doctors experienced more job stress and also burnout symptoms than doctors of colour. Depersonalisation was higher among white doctors, and there were no significant racial differences regarding EE and PA. In this study, however, some of the ethnic groups were too poorly represented to draw any definite conclusions regarding the levels of burnout by ethnicity.

Neither registrars nor medical officers were spared when it came to experiencing high degrees of burnout. This would indicate that the levels of burnout are not specifically influenced by the post description as measured in this study.

Doctors working mixed shifts showed more emotional exhaustion than those working day shifts only, which may be an indication that the loss of sleep leads not only to physical exhaustion, but also to emotional exhaustion.

This is, to the authors' knowledge, the first study investigating the prevalence of burnout among registrars and medical officers in public healthcare facilities in Bloemfontein. A similar study done in the Cape Town metropolitan area found an equal or higher degree of burnout among its participants, using the same measuring tools. ${ }^{13}$ These studies have documented that South African doctors suffer from severe burnout levels. This may well be caused by a high demand for the medical services within the context of limited professional resources available.

An action plan needs to be put in place in collaboration with the Departments of Health and Education to address burnout among this important cadre. Solutions to alleviate stress that may lead to burnout could include focusing on improving workplace conditions and circumstances for the registrars and medical 
Table 3: Distribution of participants by degree of burnout (high, moderate, low) for all three dimensions overall and according to gender and ethnicity, job roles and shifts

\begin{tabular}{|c|c|c|c|c|c|c|c|c|c|c|c|c|}
\hline \multirow{4}{*}{$\begin{array}{l}\text { Level of } \\
\text { burnout per } \\
\text { dimension }\end{array}$} & \multirow{4}{*}{$\begin{array}{l}\text { Overall } \\
\begin{array}{l}n=205 \\
n(\%)\end{array}\end{array}$} & \multicolumn{2}{|c|}{ Gender } & \multicolumn{5}{|c|}{ Ethnicity } & \multicolumn{2}{|c|}{ Job role } & \multicolumn{2}{|c|}{ Shifts worked } \\
\hline & & Male & Female & Black & White & Coloured & Indian & Other & Registrar & $\begin{array}{c}\text { Medical } \\
\text { Officer }\end{array}$ & $\begin{array}{l}\text { Day } \\
\text { shift }\end{array}$ & $\begin{array}{l}\text { Mixed } \\
\text { shifts } \\
\text { and night } \\
\text { duty }\end{array}$ \\
\hline & & $n=125$ & $n=79$ & $n=58$ & $n=122$ & $n=12$ & $n=7$ & $n=6$ & $n=167$ & $n=38$ & & $n=172$ \\
\hline & & n (\%) & $n(\%)$ & $n(\%)$ & n (\%) & $n$ (\%) & $n(\%)$ & $n(\%)$ & $n(\%)$ & $n(\%)$ & $n(\%)$ & $n(\%)$ \\
\hline \multicolumn{13}{|l|}{ Sulbscale EE } \\
\hline $\operatorname{High}(\geq 27)$ & $98(47.8)$ & $45(36.0)$ & $52(65.8)$ & $24(41.4)$ & $59(48.4)$ & $5(41.7)$ & $4(57.1)$ & $6(100.0)$ & $77(46.1)$ & $21(55.3)$ & $9(27.3)$ & $89(51.7)$ \\
\hline Moderate (17-26) & $56(27.3)$ & $39(31.2)$ & $17(21.5)$ & $21(36.2)$ & $30(24.6)$ & $4(33.3)$ & $1(14.3)$ & 0 & $44(26.4)$ & $12(31.6)$ & $11(33.3)$ & $45(26.2)$ \\
\hline Low (0-16) & $51(24.9)$ & $41(32.8)$ & $10(12.7)$ & $13(22.4)$ & $33(27.1)$ & $3(25.0)$ & $2(28.6)$ & 0 & $46(27.5)$ & $5(13.2)$ & $13(39.4)$ & $38(22.1)$ \\
\hline \multicolumn{13}{|l|}{ Subscale DP } \\
\hline High ( $\geq 13)$ & $82(40.0)$ & 42 (33.6) & $39(49.4)$ & $9(15.5)$ & $59(48.4)$ & $7(58.3)$ & $3(42.9)$ & $4(66.7)$ & $65(38.9)$ & $17(44.7)$ & $7(21.2)$ & $75(43.6)$ \\
\hline Moderate (7-12) & $65(31.7)$ & $44(35.2)$ & $21(26.6)$ & $25(43.1)$ & $35(28.7)$ & $3(25.0)$ & $1(14.3)$ & $1(16.7)$ & $57(34.1)$ & $8(21.1)$ & $13(39.4)$ & $52(30.2)$ \\
\hline Low (0-6) & $58(28.3)$ & $39(31.2)$ & $19(24.1)$ & $24(41.4)$ & $28(23.0)$ & $2(16.7)$ & $3(42.9)$ & $1(16.7)$ & $45(27.0)$ & $13(34.2)$ & $13(39.4)$ & $45(26.2)$ \\
\hline \multicolumn{13}{|l|}{ Subscale PA } \\
\hline Low $(\geq 39)$ & $38(18.5)$ & $24(19.2)$ & $13(16.5)$ & $7(12.1)$ & $26(21.3)$ & $4(33.3)$ & 0 & $1(16.7)$ & $32(19.2)$ & $6(15.8)$ & $5(15.2)$ & $33(19.2)$ \\
\hline Moderate (32-38) & $89(43.4)$ & $56(44.8)$ & $33(41.8)$ & $28(48.3)$ & $53(43.4)$ & $2(16.7)$ & $4(57.1)$ & $2(33.3)$ & $72(43.1)$ & $17(44.7)$ & 19 (57.6) & $70(40.7)$ \\
\hline High (0-31) & $78(38.1)$ & $45(36.0)$ & $33(41.8)$ & $23(39.7)$ & $43(35.3)$ & $6(50.0)$ & $3(42.9)$ & $3(50.0)$ & $63(37.7)$ & $15(39.5)$ & $9(27.3)$ & $69(40.1)$ \\
\hline
\end{tabular}

Notes: $\mathrm{EE}=$ emotional exhaustion; $\mathrm{DP}=$ depersonalisation; $\mathrm{PA}=$ personal accomplishment.

officers in the public sector. Having mechanisms to ensure that experienced doctors are retained would be beneficial. Measures improving job satisfaction, management structures and health technology could reduce stress levels together with a focus on resilience training towards resilience, after-hours work and workload, early identification and de-stigmatising and management of depression. ${ }^{8,13}$ The mismatch between expectation and practicality on the ground needs to be considered.

This study has confirmed a high level/rate of burnout among the registrar workforce in Bloemfontein. An emotionally exhausted doctor with a high rate of depersonalisation and an inability to achieve personal accomplishments is unlikely to provide a satisfactorily patient-orientated service. Policy-makers in the Bloemfontein public healthcare sector need to address the problem urgently, and develop policies and strategies for dealing with burnout as it negatively affects service delivery and the well-being of healthcare workers.

\section{Study limitations}

- The response rate of $68.3 \%$ raises the possibility that those who did not participate (31.7\%) could have scored differently, thus affecting the final results. That being said, the response rate is fairly high for this kind of research, and does engender a certain level of confidence in the results.

- The MBI-HSS is a 'gold standard' tool used for measuring burnout among the caring professions, yet the responses of the participants may have been influenced by personal and work circumstances at the time they completed the questionnaires. Nonetheless, it is the authors' opinion that the results obtained from this study reliably reflect the correct state of burnout among registrars and medical officers in the public healthcare facilities in Bloemfontein at the time.

- The demographic items asking about the number of hours worked should have been phrased better so as to clarify the different portions (normal time vs. overtime) of the work done, but this was not the main focus of the study.

- Year of service might have influenced analysis of other factors. Clinical experience prior to specialist training may be a confounding variable and may result in lower levels of burnout.

\section{Conclusion}

Burnout is a common problem among registrars and medical officers in Bloemfontein public hospitals. This may well affect the level of quality patient-centred care offered. The level of burnout as measured among doctors in training by this study is high with very few not affected at all.

\section{Recommendations}

The authors believe that those responsible for the training and employing of registrars and medical officers should recognise that burnout is an occupational disease, and should take into consideration the results of this study in order to better promote and protect the health of these healthcare professionals. Stodel and Steward-Smith ${ }^{16}$ suggested a number of different steps that can be used to deal with burnout: financially based (e.g. recruitment and retention of clinical staff) and non-financially based (e.g. mentor system). Such steps would be necessary in the local health sector to deal with the current levels of burnout. Furthermore, it would be beneficial to consider the five main themes that emerged during a study by Jenkins et al. ${ }^{18}$

This study created awareness of the levels of burnout in registrars and medical officers. Further studies should focus on the prevention, causes and treatment of burnout among healthcare workers.

\section{References}

1. Maslach C, Jackson SE. The measurement of experienced burnout. J Occup Behav. 1981;2(2):99-113.

2. Ross E, Deverell B. Psychosocial approaches to health, illness and disability. Pretoria: Van Schaik; 2003. 
3. Couper I. How to cope with stress and avoid burnout. In: Mash B, Blitz-Lindeque J, editors. South African Family Practice Manual. 2nd ed. Pretoria: Van Schaik; 2006. p. 379-380.

4. Olkinuora M, Asp S, Juntunen J, et al. Stress symptoms, burnout and suicidal thoughts in Finnish physicians. Soc Psychiatry Psychiatr Epidemiol. 1990;25(2):81-86.

5. Couper ID. Approaching burnout. S Afr Fam Pract. 2005;47(2):5-8.

6. Mateen FJ, Dorji C. Health-care worker burnout and the mental health imperative. Lancet. 2009 22;374(9690):595-597. [http://www.dx.doi. org/10.1016/S0140-6736(09)61483-5] [PMID: 19699996]

7. Salyers MP, Flanagan ME, Firmin R, et al. Clinicians' perceptions of how burnout affects their work. Psychiatr Serv. 2015;66(2):204207. [http://www.dx.doi.org/10.1176/appi.ps.201400138] [PMID: 25642617]

8. Amoafo E, Hanbali N, Patel A, et al. What are the significant factors associated with burnout in doctors? Occup Med (Lond). 2015;65(2):117-121. [http://www.dx.doi.org/10.1093/occmed/ kqu144] [PMID: 25324485].

9. Cahoon SL Burnout among Canadian physicians [research project, Master of Science in Management]. Alberta: Faculty of Management, The University of Lethbridge; 2003.

10. Toral-Villanueva R, Aguilar-Madrid G, Juárez-Pérez CA. Burnout and patient care in junior doctors in Mexico City. Occup Med (Lond). 2009;59(1):8-13. [http://dx.doi.org/10.1093/occmed/kqn122] [PMID: 18796698].

11. Maslach C, Jackson SE, Leiter MP, et al. Maslach burnout inventory sampler set manual, general survey, human services survey, educators survey, \& scoring guides. Menlo Park: Mind Garden; 1986.
12. Soler JK, Yaman H, Esteva M, et al. Burnout in European family doctors: the EGPRN study. Fam Pract. 2008;25(4):245-265. [http:// dx.doi.org/10.1093/fampra/cmn038] [PMID: 18622012].

13. Rossouw L, Seedat S, Emsley RA, et al. The prevalence of burnout and depression in medical doctors working in the Cape Town Metropolitan Municipality community healthcare clinics and district hospitals of the Provincial Government of the Western Cape: a crosssectional study. S Afr Fam Pract. 2013;55(6):567-573.

14. Peltzer K, MashegoT, Mabeba M. Short Communication: Occupational stress and burnout among South African medical practitioners. Stress \& Health. 2003;19(5):275-280. [http://dx.doi.org/10.1002/smi.982]

15. Van den Berg HS, Bester CL, van Rensburg Janse, Bonthuyzen E, et al. Burnout and compassion fatigue in professional nurses: a study in PHC facilities in the Free State, with special reference to the antiretroviral treatment programme. Bloemfontein: Centre for Health Systems Research and Development, University of the Free State; 2006.

16. Stodel JM, Stewart-Smith A. The influence of burnout on skills retention of junior doctors at Red Cross War Memorial Children's Hospital: a case study. S Afr Med J. 2011;101(2):115-118.

17. Basson MJ, Rothmann S. Sense of coherence, coping and burnout of pharmacists. S Afr J Econ Manage Sci. 2002;5(1):35-62.

18. Jenkins LS, Gunst C, Blitz J, et al. What keeps health professionals working in rural district hospitals in South Africa? Afr J Prim Health Care Fam Med. 2015;7(1), Art. \#805, 5p. [http://dx.doi.org/10.4102/ phcfm.v7i1.805] [PMID: 26245623].

Received: 29-02-2016 Accepted: 02-06-2016 